\title{
Resolvendo Equações Diferenciais pelo Método da Decomposição de Adomian
}

\author{
Solving Differential Equations Using Adomian Decomposition Method
}

\author{
Ronni Geraldo Gomes de Amorim*1,20, Diogo Filipe Sens ${ }^{2}$, Alice Fazzolino Pinto Barbosa ${ }^{2}$, \\ Vinícius Carvalho Rispoli ${ }^{1}$ \\ ${ }^{1}$ Universidade de Brasília, Instituto de Física, Brasília, DF, Brasil. \\ ${ }^{2}$ Universidade de Brasília, Faculdade Gama, Brasília, DF, Brasil.
}

Recebido em 11 de Março de 2020. Revisado em 14 de Abril de 2020. Aceito em 01 de Maio de 2020.

\begin{abstract}
A procura de solução para equações diferenciais pode ser uma das principais tarefas de um físico. Nesse sentido, quanto maior o número de métodos de solução conhecidos, mais ampla é a variedade de problemas que podem ser analisados e entendidos. Dentre as equações diferenciais, as não-lineares são as que apresentam maior complexidade de resolução e rotineiramente estão presentes na descrição de fenômenos naturais. Nessa perspectiva, este trabalho apresenta uma introdução para o Método da Decomposição de Adomian, o qual é um método analítico muito utilizado para abordar equações diferenciais não-lineares. Para exemplificar a eficácia do método de Adomian serão tratados alguns exemplos lineares e não-lineares, dentre os quais pode-se destacar: a queda livre de uma partícula sob resistência do ar, o pêndulo simples e uma equação de onda unidimensional de transporte não-uniforme.

Palavras-chave: Equações Diferenciais, Problemas Não-Lineares, Método da Decomposição de Adomian.
\end{abstract}

Searching for solution of a differential equation can be one of the main tasks of a physicist. In this sense, the greater the number of known solution methods, the wider the variety of problems that can be analyzed and understood. Among the differential equations, the non-linear equations are the ones with the greatest resolution complexity and are routinely present in the description of natural phenomena. In this perspective, this work presents an introduction to the Adomian Decomposition Method, which is an analytical method vastly used to address non-linear differential equations. To exemplify the applicability of the Adomian method, some linear and non-linear examples are treated, namely: free fall of a particle under air resistance, simple gravity pendulum and a one-dimensional non-uniform transport wave equation.

Keywords: Differential Equations, Non-linear Problems, Adomian Decomposition Method.

\section{Introdução}

Os fenômenos da natureza quando modelados geralmente são apresentados na forma de uma equação diferencial; portanto, para compreendê-los bem faz-se necessário solucionar equações diferenciais. Sendo assim, resolver equações diferenciais constitui uma importante parte do trabalho de físicos, matemáticos, engenheiros e outros profissionais. Porém, nem todas as equações diferenciais são de fácil resolução analítica, existindo aquelas cujas soluções analíticas não são conhecidas. Para analisar essa última categoria, existem diversos métodos, sejam numéricos ou analíticos, a saber: séries de potências; teoria de perturbação; método de Runge-Kutta, elementos finitos, dentre outros.

Este trabalho tem como objetivo apresentar, de forma didática, um método que pode ser utilizado na solução de equações diferenciais, sejam ordinárias ou parciais, sobretudo não-lineares. Trata-se do método da decomposição de Adomian. Este método foi proposto pelo físico

*Endereço de correspondência: ronniamorim@gmail.com
Adomian na década de 1980 [1 5 com o objetivo inicial de solucionar problemas estocásticos da física. Contudo, devido a sua praticidade, tem sido aplicado em variados problemas determinísticos e estocásticos, lineares e não-lineares, diferenciais ou algébricos, de valor inicial ou de contorno e também equações diferenciais fracionárias. O método da decomposição de Adomian pode ser encontrado na solução de problemas de várias áreas, como física 6,7 , biologia [8, 9$]$ e engenharia [10 12. Exemplos de aplicações na física e engenharia são as soluções de versões não-lineares da equação de Schrödinger [7], propagação de ondas em meios elásticos [10] e solução analítica da equação de Navier-Stokes em tempo fracionário [12. Um dos pontos chaves do método de Adomian é que ele permite a solução de equações funcionais sem a necessidade de discretização ou aproximação dos operadores. Para problemas não-lineares, por exemplo, o método mostra interessantes resultados, pois sua aplicação tem possibilitado encontrar aproximações analíticas que convergem rapidamente quando a solução do problema existe. Sendo assim, além de apresentar o passo 
a passo do método, serão feitos exemplos de aplicação do método em equações diferenciais ordinárias e parciais, lineares e não-lineares.

Nesse sentido, a apresentação deste trabalho está dividida nos seguintes tópicos. Na seção2 2 será apresentado o método da decomposição de Adomian, discutindo brevemente sobre a sua convergência. Na seção 3 serão feitos exemplos da aplicação do método de Adomian tanto para problemas lineares quanto não-lineares. Os exemplos que serão tratados são: queda de um corpo sob ação da resistência do ar, equação do pêndulo simples, equação de onda unidimensional e um problema de contorno. Por fim, serão feitas as considerações finais deste trabalho.

\section{O Método da Decomposição de Adomian}

Esta seção será destinada à descrição do método da decomposição de Adomian e também uma breve análise de convergência do mesmo. A construção da solução de uma equação diferencial será conduzida em detalhes, de forma que o leitor possa entender o passo a passo para encontrar suas próprias soluções.

\subsection{Descrição do método}

Considere a seguinte equação diferencial

$$
\mathbf{F} y(x)=g(x) \text {, }
$$

em que $\mathbf{F}=\mathbf{L}+\mathbf{R}+\mathbf{N}$ representa um operador diferencial constituído por uma parte linear, na forma $\mathbf{L}+\mathbf{R}$, uma parte não linear $\mathbf{N}$, e $g(x)$ representa uma função da variável independente $x \in D \subset \mathbb{R}$. Na decomposição do operador linear, o termo $\mathbf{L}$ representa a derivada de ordem mais alta da equação diferencial equação (1) e o termo $\mathbf{R}$ representa é o restante da parte linear. Com o objetivo de exemplificar tal decomposição, considere a seguinte equação diferencial ordinária não-linear

$$
y^{\prime \prime}-2 y^{\prime}+x^{2} y+y^{3}=x^{3},
$$

em que $y=y(x)$. Na equação (2) identificam-se os termos dos operadores linear e não-linear como $\mathbf{L}=\frac{d^{2}}{d x^{2}}$ (o termo de ordem mais alta), $\mathbf{R}=-2 \frac{d}{d x}+x^{2}$ (o restante do termo linear), $\mathbf{N}=y^{3}$ (o termo não-linear) e $g(x)=x^{3}$.

Assim, a equação (1) pode ser reescrita como

$$
\mathbf{L} y(x)+\mathbf{R} y(x)+\mathbf{N} y(x)=g(x) .
$$

O primeiro passo da construção da solução é isolar a parte do operador $\mathbf{L}$ na equação (3), ou seja,

$$
\mathbf{L} y(x)=g(x)-\mathbf{R} y(x)-\mathbf{N} y(x) .
$$

No método da decomposição de Adomian o operador diferencial L deve ser inversível. Assim, aplicando o operador inverso $\mathbf{L}^{-1}$ em ambos os lados da equação (4), obtém-se que

$$
\mathbf{L}^{-1}[\mathbf{L} y(x)]=\mathbf{L}^{-1}[g(x)]-\mathbf{L}^{-1}[\mathbf{R} y(x)]-\mathbf{L}^{-1}[\mathbf{N} y(x)] .
$$

Nos casos trabalhados neste artigo, considerando que as equações aqui envolvidas são diferenciais, o operador inverso $\mathbf{L}^{-1}$ corresponde a uma integral repetida na variável independente, em que o número de repetições é exatamente àquele da ordem da derivada de L. Suponha, por exemplo, que o lado esquerdo da equação (4) seja um operador correspontente a uma derivada de primeira ordem, ou seja, $\mathbf{L}=\frac{d}{d x}$, neste caso o operador inverso será dado por $\mathbf{L}^{-1}=\int_{0}^{x}(\cdot) d s$.

Reescrevendo a equação (5) tem-se

$$
y(x)=\varphi+\mathbf{L}^{-1}[g(x)]-\mathbf{L}^{-1}[\mathbf{R} y(x)]-\mathbf{L}^{-1}[\mathbf{N} y(x)],
$$

em que $\varphi$ é uma constante que surge da aplicação do operador inverso e está relacionado as condições iniciais.

As principais hipóteses do método da decomposição de Adomian [1 5] são admitir que a solução $y(x)$ possa ser decomposta como uma série de funções, isto é,

$$
y(x)=\sum_{n=0}^{\infty} y_{n}(x)
$$

e que o termo não-linear $\mathbf{N} y(x)$ seja uma função analítica podendo ser escrita por meio dos denominados polinômios de Adomian $A_{n}(x)$ na forma

$$
\mathbf{N} y(x)=\sum_{n=0}^{\infty} A_{n}(x) .
$$

Desta forma, substituindo as equações (7) e (8) na equação (6) tem-se que

$$
\begin{aligned}
\sum_{n=0}^{\infty} y_{n}(x) & =\varphi+\mathbf{L}^{-1}[g(x)]-\mathbf{L}^{-1}\left[\sum_{n=0}^{\infty} \mathbf{R}\left(y_{n}(x)\right)\right] \\
& -\mathbf{L}^{-1}\left[\sum_{n=0}^{\infty} A_{n}(x)\right]
\end{aligned}
$$

Neste contexto, cada parcela da série infinita, dada pela equação (7), $y_{0}(x), y_{1}(x), y_{2}(x), \cdots, y_{n}(x), \cdots$ pode ser calculada a partir da equação 9 por comparação direta entre os lados direito e esquerdo da equação. Fazendo isso, obtem-se o seguinte algoritmo para a solução

$$
\begin{aligned}
y_{0}(x)= & \varphi+\mathbf{L}^{-1}[g(x)] \\
y_{n+1}(x)= & -\mathbf{L}^{-1}\left(A_{n}(x)+\mathbf{R}\left(y_{n}(x)\right),\right. \\
& n=0,1,2, \cdots
\end{aligned}
$$

Note que a solução é obtida de forma iterativa pelas equações (10) e (11). Além disso, os termos da série $\sum y_{n}(x)$ dependem diretamente dos polinômios de Adomian. Para se construir os polinômios de Adomian deve-se fazer a expansão em série de Taylor para a função não-linear $\mathbf{N} y(x)$ em torno da função inicial $y_{0}(x)$ [1 $\left[\begin{array}{l}5] \\ \text {. }\end{array}\right.$ 
Como por hipótese a solução da equação (1) é na forma $y(x)=\sum_{n=0}^{\infty} y_{n}(x)$ e o termo não linear pode ser escrito na forma $\mathbf{N} y(x)=\sum_{n=0}^{\infty} A_{n}(x)$, então através de um longo processo de comparação entre duas séries obtem-se os polinômios de Adomian [1 5]. Através deste processo é possível mostrar que a fórmula geral para o cálculo dos polinômios $A_{n}$ 's é dada por 1,5

$$
A_{n}(x)=\frac{1}{n !} \frac{d^{n}}{d \lambda^{n}}\left[\mathbf{N} \sum_{i=0}^{n} y_{i}(x) \lambda^{i}\right]_{\lambda=0}, n=0,1,2, \cdots
$$

Neste caso, para a utilização nos exemplos que serão apresentados na Seção 3 traz-se os cinco primeiros polinômios calculados a partir da equação 12

$$
\begin{aligned}
A_{0}(x) & =\mathbf{N} y_{0}(x) \\
A_{1}(x) & =y_{1}(x) \mathbf{N}^{\prime} y_{0}(x) \\
A_{2}(x) & =\frac{y_{1}^{2}(x)}{2} \mathbf{N}^{\prime \prime} y_{0}(x)+y_{2}(x) \mathbf{N}^{\prime} y_{0}(x) \\
A_{3}(x) & =y_{3}(x) \mathbf{N}^{\prime} y_{0}(x)+y_{1}(x) y_{2}(x) \mathbf{N}^{\prime \prime} y_{0}(x) \\
& +\frac{y_{1}(x)^{3}}{3 !} \mathbf{N}^{\prime \prime \prime} y_{0}(x) \\
A_{4}(x) & =y_{4}(x) \mathbf{N}^{\prime} y_{0}(x) \\
& +\left(y_{1}(x) y_{3}(x)+\frac{y_{2}(x)^{2}}{2}\right) \mathbf{N}^{\prime \prime} y_{0}(x) \\
& +\frac{y_{1}(x)^{2} y_{2}(x)}{2} \mathbf{N}^{\prime \prime \prime} y_{0}(x) \\
& +\frac{y_{1}(x)^{4}}{4 !} \mathbf{N}^{(4)} y_{0}(x)
\end{aligned}
$$

Dado o algoritmo para construção da solução de uma equação diferencial, a seguir será feita uma breve análise sobre a a convergência do método de Adomian apresentado.

\subsection{Sobre a Convergência do Método}

Nesta subseção será feita uma breve discussão sobre a convergência do método de Adomian. Isto ajudará na verificação da viabilidade do método em determinadas situações.

Considerando de forma geral a equação funcional

$$
y-\mathbf{N} y=f,
$$

em que $\mathbf{N}: H \rightarrow H$ é um operador não-linear contínuo, $f \in H$ e $H$ é um espaço de Hilbert, i.e., são espaços vetoriais com produto interno e completos 12 13. O objetivo do método da decomposição de Adomian é buscar uma função $y \in H$ tal que a equação (18) seja satisfeita. Isto é feito supondo que a função $y$ possa ser escrita como uma série

$$
y(x)=\sum_{n=0}^{\infty} y_{n}(x)
$$

e também que o termo não-linear possa ser escrito na forma

$$
\mathbf{N} y(x)=\sum_{n=0}^{\infty} A_{n}(x)
$$

Conforme descrito na Subseção 2.1. o método levará a seguinte relação de recorrência

$$
\left\{\begin{array}{l}
y_{0}(x)=f(x) \\
y_{k+1}(x)=A_{k}\left(y_{0}(x), y_{1}(x), \cdots, y_{k}(x)\right)
\end{array}\right.
$$

Diante disto é possível enunciar o seguinte teorema sobre a convergência do método da decomposição de Adomian. Para o leitor interessado a demonstração deste teorema pode ser encontrado na referência 14 . Se $\mathbf{N}$ : $H \rightarrow H$ é um operador contínuo no espaço de Hilbert $H$ e $y(x)$ é a solução da equação 18 , então a série $\sum_{n=0}^{\infty} y_{n}(x)$, cujos termos são obtidos pela relação de recorrência (21), converge para $y(x)$ sempre que existir $0 \leq \alpha<1$ e $n_{0} \in \mathbb{N}$ satisfazendo $\left\|y_{k+1}\right\|_{\infty} \leq \alpha\left\|y_{k}\right\|_{\infty}$, para todo $k \geq n_{0}[14$.

Este resultado oferece uma forma simples verificar se o método irá convergir para um determinado tipo de problema. Como é possível verificar na literatura, nem sempre o método da decomposição de Adomian descrito neste trabalho resultará em séries convergentes [14]. Neste caso, duas opções são possíveis: ou trabalhar com alguma forma modificada do método da decomposição de Adomian [15] ou fazer a escolha de um método numérico diferente.

Na literatura há diversos outros resultados e generalizações referentes à análise de convergência do método da decomposição de Adomian, o leitor interessado pode consultar as referências $[1622$ para maiores detalhes.

\section{Aplicações do Método da Decomposição de Adomian}

Nesta seção o objetivo é exemplificar o uso do método da decomposicão de Adomian através de algumas equações comuns na física-matemática. Serão construídas soluções analíticas e semi-analíticas de equações diferenciais ordinárias não-lineares e também um exemplo de uma equação diferencial parcial.

\subsection{Queda de um corpo sob resistência do ar}

O primeiro problema que será apresentado trata do movimento vertical de queda livre de um corpo nas proximidades da Terra levando em consideração a resistência do ar. O interessante deste problema é que ele possui solução analítica de construção trivial e, desta forma, ela será comparada com a solução obtida utilizando o método da decomposição de Adomian. Observa-se nessa situação que o objetivo deste exemplo é apenas para ilustrar o método da decomposição de Adomian, afinal neste caso é mais simples obter a solução analítica através do método da separação de variáveis do que através do método de Adomian.

Considere que a aceleração gravitacional seja constante e igual a $g$. Além disso, considere que a força de resistência do ar seja dada por $F_{a r}=\frac{1}{2} C \rho A v^{2}$, em que $C$ represente 
o coeficiente de arrasto, $\rho$ a densidade do ar, e $A$ a área da seção frontal do corpo em queda 23]. Além disso, suponha que esse corpo tenha massa $m$ e que sua velocidade inicial seja nula, ou seja, $v(0)=0$. Desta forma, pela segunda lei de Newton tem-se a seguinte equação diferencial de primeira ordem não-linear

$$
m \frac{d v}{d t}=m g-\frac{1}{2} C \rho A v^{2},
$$

que reescrevendo assume a forma

$$
\frac{d v}{d t}=g-\frac{C \rho A}{2 m} v^{2} .
$$

A equação (3.1.2 é separável e é possível calcular a solução analítica do problema de valor inicial de forma direta através do método das frações parciais obtendo a seguinte expressão

$$
v(t)=\sqrt{\frac{2 g m}{C \rho A}} \tanh \left(\sqrt{\frac{g C \rho A}{2 m}} t\right) .
$$

O objetivo é comparar a solução analítica com a solução obtida através do método da decomposição de Adomian. Desta forma, aplicando o método descrito na Seção 2 . pode-se escrever a equação (3.1.2 na forma

$$
\mathbf{L} v(t)=g-\frac{C \rho A}{2 m} v(t)^{2},
$$

em que $\mathbf{L}=\frac{d}{d t}$ representa o operador diferencial e $\mathbf{N} v=\frac{C \rho A}{2 m} v^{2}$ representa o termo não-linear. Utilizando o operador inverso $\mathbf{L}^{-1}=\int_{0}^{t}(\cdot) d s$ em ambos os lados da equação 3.1.4 obtem-se

$$
\begin{aligned}
\int_{0}^{t} \frac{d v}{d t} d s & =\int_{0}^{t} g d s-\frac{C \rho A}{2 m} \int_{0}^{t} v^{2}(s) d s \Rightarrow \\
v(t)-v(0) & =g t-\frac{C \rho A}{2 m} \int_{0}^{t} v^{2}(s) d s
\end{aligned}
$$

Aqui supõe-se que a solução $v(t)$ seja dada por uma série de funções $v(t)=\sum_{n=0}^{\infty} v_{n}(t)$ e que o termo não-linear pode ser escrito por meio de uma série dos polinômios de Adomian, $\mathbf{N} v=\sum_{n=0}^{\infty} A_{n}(t)$. Logo, a equação 3.1.5 será reescrita na forma

$$
\sum_{n=0}^{\infty} v_{n}(t)=g t-\int_{0}^{t} \sum_{n=0}^{\infty} A_{n}(s) d s,
$$

lembrando que o corpo inicialmente está em repouso, i.e., $v(0)=0$. Portanto, a equação 3.1.6 leva à seguinte relação de recorrência

$$
\begin{aligned}
v_{0}(t) & =g t \\
v_{n+1}(t) & =-\int_{0}^{t} A_{n}(s) d s .
\end{aligned}
$$

Da equação (3.1.7), verifica-se de imediato, através da equação 13 , que o polinômio $A_{0}(t)$ é dado por

$$
\begin{aligned}
A_{0}(t) & =\frac{C \rho A}{2 m}\left(v_{0}\right)^{2} \\
& =\frac{g^{2} C \rho A}{2 m} t^{2} .
\end{aligned}
$$

Em seguida, tem-se que o termo $v_{1}(t)$ da série é calculado pela equação 3.1.8 que é dado por

$$
\begin{aligned}
v_{1}(t) & =-\int_{0}^{t} A_{0}(s) d s \\
& =-\frac{g^{2} C \rho A}{6 m} t^{3} .
\end{aligned}
$$

Para o cálculo do próximo termo $v_{2}(t)$ da série, deve-se calcular o próximo polinômio de Adomian $A_{1}(t)$ que é dado pela equação 14 , isto é,

$$
\begin{aligned}
A_{1}(t) & =v_{1}(t) \mathbf{N}^{\prime} v_{0}(t) \\
& =\frac{C \rho A}{m} v_{1}(t) v_{0}(t) \\
& =-\frac{g^{3} C^{2} \rho^{2} A^{2}}{6 m^{2}} t^{4}
\end{aligned}
$$

Assim, o termo $v_{2}(t)$ é calculado como sendo

$$
\begin{aligned}
v_{2}(t) & =-\int_{0}^{t} A_{1}(s) d s \\
& =\frac{g^{3} C^{2} \rho^{2} A^{2}}{30 m^{2}} t^{5} .
\end{aligned}
$$

Repetindo esse procedimento para o termo $v_{3}(t)$, primeiramente deve-se calcular o polinômio $A_{3}(t)$ que é dado pela equação 15 que é dado por

$$
\begin{aligned}
A_{2}(t) & =v_{2}(t) \mathbf{N}^{\prime} v_{0}(t)+\frac{v_{1}(t)^{2}}{2} \mathbf{N}^{\prime \prime} v_{0}(t) \\
& =\frac{C \rho A}{2 m}\left[2 v_{0}(t) v_{2}(t)+v_{1}(t)^{2}\right] \\
& =\frac{17}{360} \frac{g^{4} C^{3} \rho^{3} A^{3}}{m^{3}} t^{6}
\end{aligned}
$$

Logo, o termo $v_{3}(t)$ pode ser calculado e é dado por

$$
\begin{aligned}
v_{3}(t) & =-\int_{0}^{t} A_{2}(s) d s \\
& =-\frac{17}{2520} \frac{g^{4} C^{3} \rho^{3} A^{3}}{m^{3}} t^{7} .
\end{aligned}
$$

Finalmente, tem-se os quatro primeiros termos da solução que é dada por

$$
\begin{aligned}
v(t) & =g t-\frac{g^{2} C \rho A}{6 m} t^{3}+\frac{g^{3} C^{2} \rho^{2} A^{2}}{30 m^{2}} t^{5} \\
& -\frac{17}{2520} \frac{g^{4} C^{3} \rho^{3} A^{3}}{m^{3}} t^{7}+\cdots
\end{aligned}
$$

É possível de forma direta, apesar de não imediata, reescrever a série encontrada na equação 3.1.15 na seguinte 
forma

$$
\begin{aligned}
& v(t)=\sqrt{\frac{2 g m}{C \rho A}}\left[\left(\sqrt{\frac{g C \rho A}{2 m}} t\right)-\frac{1}{3}\left(\sqrt{\frac{g C \rho A}{2 m}} t 3.1 .16\right)\right. \\
& \left.+\frac{2}{15}\left(\sqrt{\frac{g C \rho A}{2 m}} t\right)^{5}-\frac{17}{315}\left(\sqrt{\frac{g C \rho A}{2 m}} t\right)^{7}+\cdots\right] .
\end{aligned}
$$

Observe que a série que aparece na solução dada pela equação (3.1.16 é exatamente a expansão em série de Taylor da função tanh $\left(\sqrt{\frac{g C \rho A}{2 m}} t\right)$. Portanto, a solução encontrada utilizando método da decomposição de Adomian corresponde àquela que podemos calcular diretamente resolvendo a equação diferencial separável.

\subsection{Equação do pêndulo simples}

Outro sistema físico bastante conhecido e cuja análise recai numa equação diferencial não-linear de segunda ordem é o pêndulo simples não-amortecido. A equação diferencial que descreve o movimento de um pêndulo de comprimento $l$ apenas sobre a ação da gravidade $g$ é dada pela equação 24

$$
\frac{d^{2} \theta(t)}{d t^{2}}+\omega_{0}^{2} \sin (\theta)=0
$$

em que $\theta$ representa o ângulo que o pêndulo forma com o eixo vertical, $t$ representa o tempo e $\omega_{0}=\sqrt{g / l}$. Além disso, suponha que as condições iniciais sejam dadas por

$$
\begin{aligned}
\theta(0) & =\varphi_{0} \\
\left(\frac{d \theta}{d t}\right)_{t=0} & =0 .
\end{aligned}
$$

Observa-se que a equação 3.2.1, sujeita às condições iniciais 3.2.2 e 3.2.3), possui solução analítica dada por

$$
\begin{array}{r}
\theta(t)=2 \arcsin \left\{\operatorname { s i n } ( \frac { \varphi _ { 0 } } { 2 } ) \operatorname { s n } \left[K\left(\sin ^{2}\left(\frac{\varphi_{0}}{2}\right)\right)-\right.\right. \\
\left.\left.\omega_{0} t ; \sin ^{2}\left(\frac{\varphi_{0}}{2}\right)\right]\right\}
\end{array}
$$

em que $\operatorname{sn}(v ; w)$ representa as funções elípticas de Jacobi e $K(v)$ é a integral elíptica completa de primeira espécie 24.

$\mathrm{Na}$ equação 3.2.1 observa-se que o operador linear é dado por $\mathbf{L}=\frac{d^{2}}{d t^{2}}$, assim como a parte não-linear é dada por $\mathbf{N} \theta=\sin (\theta)$. Para utilizar o método da decomposição de Adomian, tem-se que o operador inverso é dado, neste caso, por $\mathbf{L}^{-1}=\int_{0}^{t} \int_{0}^{s}(\cdot) d u d s$. Aplicando o operador linear inverso, $\mathbf{L}^{-1}$ em ambos os membros da equação (3.2.1), tem-se

$$
\begin{aligned}
& \mathbf{L}^{-1} \mathbf{L} \theta(t)=-\omega_{0}^{2} \mathbf{L}^{-1} \mathbf{N} \theta \Rightarrow \\
& \int_{0}^{t} \int_{0}^{s} \frac{d^{2} \theta(u)}{d u^{2}} d u d s=-\omega_{0}^{2} \mathbf{L}^{-1}[\sin (\theta)] \Rightarrow \\
& \theta(t)-\theta(0)-\frac{d \theta(0)}{d t} t=-\omega_{0}^{2} \mathbf{L}^{-1}[\sin (\theta)],(3
\end{aligned}
$$

ou seja,

$$
\theta(t)=\varphi_{0}-\omega_{0}^{2} \mathbf{L}^{-1}[\sin (\theta)]
$$

O próximo passo do processo de solução é supor que a solução da equação do pêndulo $\theta(t)$ seja dada por uma série de funções de $t$, ou seja, $\theta(t)=\sum_{n=0}^{\infty} \theta_{n}(t)$; além disso, deve-se supor que o termo não-linear, $\sin (\theta)$, possa ser escrito em termos dos polinômios de Adomian $\mathbf{N} \theta=$ $\sin (\theta)=\sum_{n=0}^{\infty} A_{n}(t)$. Considerando essas suposições, tem-se que a equação 3.2 .6 pode ser reescrita como

$$
\sum_{n=0}^{\infty} \theta_{n}(t)=\varphi_{0}-\omega_{0}^{2} \mathbf{L}^{-1}\left[\sum_{n=0}^{\infty} A_{n}(t)\right]
$$

Comparando ambos os lados da equação (3.2.7), identificase a seguinte relação de recorrência:

$$
\begin{aligned}
\theta_{0}(t) & =\varphi_{0} \\
\theta_{n+1}(t) & =-\omega_{0}^{2} \mathbf{L}^{-1}\left[A_{n}(t)\right]
\end{aligned}
$$

De acordo com a equação 13 , tem-se que o primeiro polinômio de Adomian é dado por

$$
\begin{aligned}
A_{0}(t) & =\mathbf{N} \theta_{0} \\
& =\sin \left(\varphi_{0}\right) .
\end{aligned}
$$

Com isso é possível calcular a parcela $\theta_{1}(t)$ da série de solução como

$$
\begin{aligned}
\theta_{1}(t) & =-\omega_{0}^{2} \mathbf{L}^{-1}\left[A_{0}(t)\right] \\
& =-\omega_{0}^{2} \mathbf{L}^{-1}\left[\sin \left(\varphi_{0}\right)\right] \\
& =-\sin \left(\varphi_{0}\right) \frac{\left(\omega_{0} t\right)^{2}}{2}
\end{aligned}
$$

A partir das funcões $\theta_{0}(t)$ e $\theta_{1}(t)$, pode-se calcular o polinômio de Adomian $A_{1}(t)$ por meio da equação (14). Neste caso, tem-se que

$$
\begin{aligned}
A_{1}(t) & =\theta_{1}\left(\mathbf{N}^{\prime} \theta\right)_{\theta=\theta_{0}} \\
& =\theta_{1}\left(\frac{d}{d \theta} \sin (\theta)\right)_{\theta=\theta_{0}} \\
& =-\sin \left(\varphi_{0}\right) \cos \left(\varphi_{0}\right) \frac{\left(\omega_{0} t\right)^{2}}{2}
\end{aligned}
$$

Assim, é imediato calcular o termo $\theta_{2}(t)$ da série de solução que é dado por

$$
\begin{aligned}
\theta_{2}(t) & =-\omega_{0}^{2} \mathbf{L}^{-1}\left[A_{1}\right] \\
& =-\omega_{0}^{2} \mathbf{L}^{-1}\left[-\sin \left(\varphi_{0}\right) \cos \left(\varphi_{0}\right) \frac{\left(\omega_{0} t\right)^{2}}{2}\right] \\
& =\sin \left(\varphi_{0}\right) \cos \left(\varphi_{0}\right) \frac{\left(\omega_{0} t\right)^{4}}{4 !}
\end{aligned}
$$


Agora, calculando o polinômio $A_{2}(t)$ a partir da equação 15 tem-se

$$
\begin{aligned}
A_{2}(t) & =\theta_{2}\left(\mathbf{N}^{\prime} \theta\right)_{\theta=\theta_{0}}+\frac{\theta_{1}^{2}}{2}\left(\mathbf{N}^{\prime \prime} \theta\right)_{\theta=\theta_{0}} \\
& =\theta_{2}\left(\frac{d}{d \theta} \sin (\theta)\right)_{\theta=\theta_{0}}+\frac{\theta_{1}^{2}}{2 !}\left(\frac{d^{2}}{d \theta^{2}} \sin (\theta)\right)_{\theta=\theta_{0}} \\
& =\theta_{2} \cos \left(\theta_{0}\right)-\frac{\theta_{1}^{2}}{2} \sin \left(\theta_{0}\right) \\
& =\left(\sin \left(\varphi_{0}\right)-4 \sin ^{3}\left(\varphi_{0}\right)\right) \frac{\left(\omega_{0} t\right)^{4}}{4 !} .
\end{aligned}
$$

E assim, o quarto termo da série de solução é dado por

$$
\begin{aligned}
\theta_{3}(t) & =-\omega_{0}^{2} \mathbf{L}^{-1}\left[A_{2}\right] \\
& =-\omega_{0}^{2} \mathbf{L}^{-1}\left[\left(\sin \left(\varphi_{0}\right)-4 \sin ^{3}\left(\varphi_{0}\right)\right) \frac{\left(\omega_{0} t\right)^{4}}{4 !}\right] \\
& =-\left(\sin \left(\varphi_{0}\right)-4 \sin ^{3}\left(\varphi_{0}\right)\right) \frac{\left(\omega_{0} t\right)^{6}}{6 !}
\end{aligned}
$$

É possível repetir esse processo até obter a precisão desejada. Neste caso, tem-se a série da solução $\theta(t)$ até a quarta parcela dada por

$$
\begin{aligned}
\theta(t) & =\varphi_{0}-\sin \left(\varphi_{0}\right) \frac{\left(\omega_{0} t\right)^{2}}{2 !}+\sin \left(\varphi_{0}\right) \cos \left(\varphi_{0}\right) \frac{\left(\omega_{0} t\right)^{4}}{4 !} \\
& -\left(\sin \left(\varphi_{0}\right)-4 \sin ^{3}\left(\varphi_{0}\right)\right) \frac{\left(\omega_{0} t\right)^{6}}{6 !}+\cdots \quad(3.2 .16)
\end{aligned}
$$

Na figura 1 é possível ver uma comparação entre a solução analítica dada pela equação (3.2.4) e a solução aproximada como a obtida na equação (3.2.16), para $\varphi_{0}=\pi / 8$ e $\omega_{0}=1 / 8$. A solução aproximada considerada na figura 1 foi calculada usando uma série com seis termos. Verifica-se qualitativamente que a solução aproximada está de acordo com a solução analítica, fato que é validado com o auxílio da figura 2 . Nota-se em ambas as figuras que o erro absoluto aumenta consideravalmente na parte direita do intervalo no qual a solução é calculada. Porém, é possível verificar que no pior caso a solução aproximada

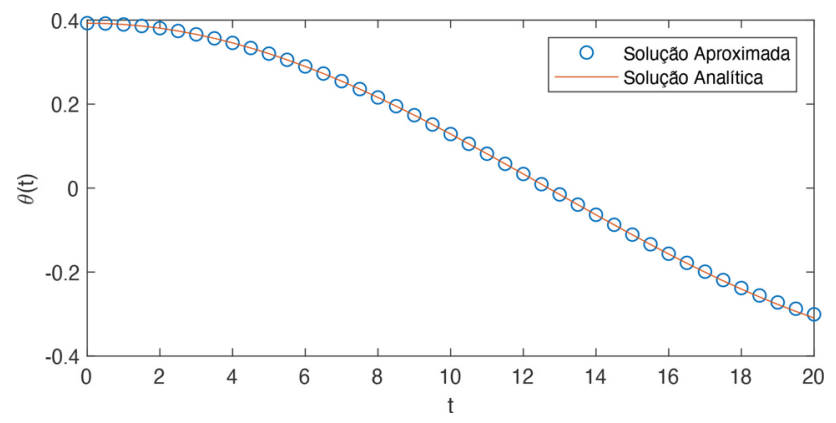

Figura 1: Comparação entre a solução analítica, equação 3.2.4, e a solução aproximada, equação (3.2.16), obtida através do método da decomposição de Adomian considerando $\varphi_{0}=\pi / 8 \mathrm{e}$ $\omega_{0}=1 / 8$. A solução aproximada contém até a sexta parcela da série e mostra concordância com a solução analítica no intervalo considerado.

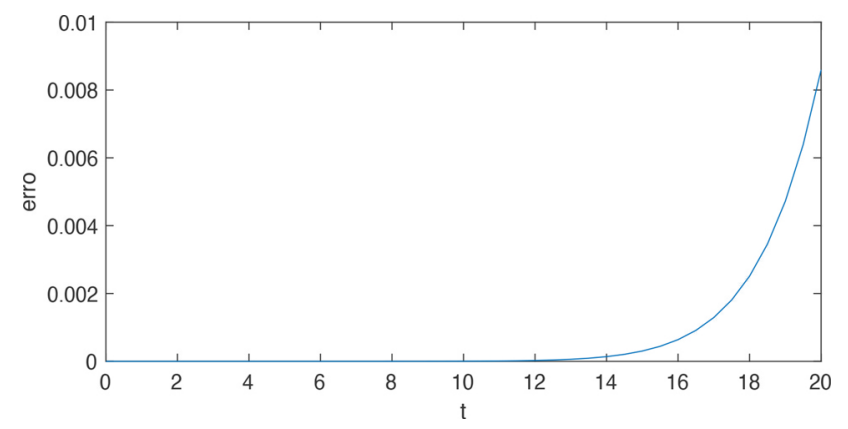

Figura 2: Erro absoluto entre a solução analítica, equação 3.2.4 , e a solução aproximada, equação 3.2 .16 , para $\varphi_{0}=$ $\pi / 8$ e $\omega_{0}=1 / 8$. No intervalo considerado, a solução aproximada tem duas casas decimais de precisão.

ainda possui precisão de duas casas decimais. Para que seja possível aumentar a precisão ou estender o intervalo de análise seria preciso aumentar o número de parcelas na série aproximada calculada.

\subsection{Equação da onda unidimensional}

O método da decomposição de Adomian também pode ser útil para se resolver equações diferenciais parciais. Além disso, será verificado que este método permite tratar equações diferenciais lineares e não apenas equações nãolineares. Nesta seção será apresentada a solução de um problema de Cauchy para uma equação de transporte não-homogêneo.

Considere o problema o problema de Cauchy

$$
\begin{cases}\frac{\partial u}{\partial t}-x \frac{\partial u}{\partial x}=0, & (x, t) \in \mathbb{R} \times \mathbb{R}_{\geq 0} \\ u(x, 0)=1-x^{2}, & x \in \mathbb{R}\end{cases}
$$

observe que a equação diferencial do problema (3.3.1 é linear. Além disso, este problema de valor inicial pode ser resolvido analiticamente através do método das características 25]. Neste caso, a solução é dada pela a função $u(x, t)=1-x^{2} e^{2 t}$.

Aqui o operador linear é dado por $\mathbf{L}=\frac{\partial}{\partial t}$, assim o operador inverso pode ser escrito como $\mathbf{L}^{-1}=\int_{0}^{t}(\cdot) d s$. Aplicando o operador inverso na equação diferencial, tem-se

$$
\begin{aligned}
\mathbf{L}^{-1} \mathbf{L} u(x, t) & =\mathbf{L}^{-1} x \frac{\partial}{\partial x} u(x, t) \Rightarrow \\
\int_{0}^{t} \frac{\partial}{\partial s} u(x, s) d s & =\int_{0}^{t} x \frac{\partial}{\partial x} u(x, s) d s \Rightarrow \\
u(x, t)-u(x, 0) & =x \int_{0}^{t} \frac{\partial}{\partial x} u(x, s) d s,
\end{aligned}
$$

ou seja,

$$
u(x, t)=\left(1-x^{2}\right)+x \int_{0}^{t} \frac{\partial}{\partial x} u(x, s) d s .
$$


Supondo agora que a função $u(x, t)$ possa ser escrita como uma série de funções de $u(x, t)$, isto é, $u(x, t)=$ $\sum_{n=0}^{\infty} u_{n}(x, t)$, então substituindo na equação 3.3 .2 obtem-se

$$
\sum_{n=0}^{\infty} u_{n}(x, t)=\left(1-x^{2}\right)+x \sum_{n=0}^{\infty} \int_{0}^{t} \frac{\partial}{\partial x} u_{n}(x, s) d s .
$$

Observe que neste caso não há termo não-linear, desta forma os polinômios de Adomian não serão necessários no processo de solução.

Ao comparar os dois lados da equação 3.3.3 identificase a relação de recorrência

$$
\begin{aligned}
u_{0}(x, t) & =1-x^{2} \\
u_{n+1}(x, t) & =x \int_{0}^{t} \frac{\partial}{\partial x} u_{n}(x, s) d s .
\end{aligned}
$$

Assim, é possível encontrar as funções $u_{n}(x, t)$ começando por $u_{1}(x, t)$ que é dado por

$$
\begin{aligned}
u_{1}(x . t) & =x \int_{0}^{t} \frac{\partial}{\partial x} u_{0}(x, s) d s \\
& =x \int_{0}^{t} \frac{\partial}{\partial x}\left(1-x^{2}\right) d s \\
& =-2 x^{2} t .
\end{aligned}
$$

Em seguida, pode-se calcular a função $u_{2}(x, t)$ que é dada por

$$
\begin{aligned}
u_{2}(x . t) & =x \int_{0}^{t} \frac{\partial}{\partial x} u_{1}(x, s) d s \\
& =x \int_{0}^{t} \frac{\partial}{\partial x}\left(-2 x^{2} t\right) d s \\
& =-x^{2} \frac{(2 t)^{2}}{2 !} .
\end{aligned}
$$

Para o quarto termo da soma, tem-se

$$
\begin{aligned}
u_{3}(x . t) & =x \int_{0}^{t} \frac{\partial}{\partial x} u_{2}(x, s) d s \\
& =x \int_{0}^{t} \frac{\partial}{\partial x}\left(-4 x^{2} t^{2}\right) d s \\
& =-x^{2} \frac{(2 t)^{3}}{3 !} .
\end{aligned}
$$

Neste caso, é imediato mostrar, por indução, que para $\begin{array}{lllll}\text { todo } & n & \mathbb{N} & \end{array}$ $(n+1)$-ésimo termo da soma é

$$
u_{n}(x, t)=-x^{2} \frac{(2 t)^{n}}{n !}
$$

Portanto, tem-se a solução dada pelo método da decomposição de Adomian

$$
\begin{aligned}
u(x, t) & =\sum_{n=0}^{\infty} u_{n}(x, t) \\
& =\left(1-x^{2}\right)-\sum_{n=1}^{\infty} x^{2} \frac{(2 t)^{n}}{n !} \\
& =1-x^{2} \sum_{n=0}^{\infty} \frac{(2 t)^{n}}{n !} \\
& =1-x^{2} e^{2 t}
\end{aligned}
$$

que coincide com a forma fechada da solução do problema 3.3.1.

\subsection{Problema de contorno}

Finalmente, neste último exemplo, será verificado que o método da decomposição de Adomian também pode ser utilizado para se resolver problemas de valor de contorno. Para tal considere a equação diferencial linear

$$
\frac{d^{2} y}{d x^{2}}=y
$$

sujeita às condições de contorno $y(0)=0$ e $y(1)=1$. A solução para este problema é conhecida e dada por

$$
y(x)=\operatorname{csch}(1) \sinh (x) .
$$

O operador linear associado a esse problema é de segunda ordem e dado por $\mathbf{L}=\frac{d^{2}}{d x^{2}}$, assim será escolhido o operador inverso escrito na forma $\mathbf{L}^{-1}=\int_{x}^{1}\left(\int_{0}^{s}(\cdot) d u\right) d s$. É importante notar que há diferentes escolhas para o operador inverso, porém é possível provar que a solução obtida será a mesma em todos os casos 26 28.

Aplicando o operador inverso na equação diferencial, tem-se

$$
\begin{aligned}
& \mathbf{L}^{-1} \mathbf{L} y(x)=\mathbf{L}^{-1} y(x) \Rightarrow \\
& \int_{x}^{1} \int_{0}^{s} \frac{d^{2} y}{d u^{2}} d u d s=\int_{x}^{1} \int_{0}^{s} y(u) d u d s \Rightarrow \\
& y(1)-y(x)+(1-x) y^{\prime}(0)=\int_{x}^{1} \int_{0}^{s} y(u) d u d(3.4 .3)
\end{aligned}
$$

e, portanto,

$$
y(x)=1+(x-1) y^{\prime}(0)-\int_{x}^{1} \int_{0}^{s} y(u) d u d s .
$$

$\mathrm{Na}$ aplicação do operador diferencial surgiu o termo desconhecido $y^{\prime}(0)$ e para o processo de obtenção da solução continuar é preciso calcular esse termo. Como $y(0)=0$ é conhecido, então fazendo $x=0$ na equação (3.4.4 tem-se

$$
y^{\prime}(0)=1-\int_{0}^{1} \int_{0}^{s} y(u) d u d s .
$$


Portanto,

$y(x)=x-(x-1) \int_{0}^{1} \int_{0}^{s} y(u) d u d s-\int_{x}^{1} \int_{0}^{s} y(u) d u d s$.

Supondo agora que $y(x)=\sum_{n=0}^{\infty} y_{n}(x)$, então substituindo na equação (3.4.6) obtem-se

$$
\begin{aligned}
\sum_{n=0}^{\infty} y_{n}(x) & =x-(x-1) \sum_{n=0}^{\infty} \int_{0}^{1} \int_{0}^{s} y_{n}(u) d u d s \\
& -\sum_{n=0}^{\infty} \int_{x}^{1} \int_{0}^{s} y_{n}(u) d u d s .
\end{aligned}
$$

Novamente tem-se uma equação linear, logo os polinômios de Adomian não surgirão no processo de solução. Assim, comparando os lados direito e esquerdo da equação (3.4.7) identifica-se a seguinte relação de recorrência

$$
\begin{aligned}
y_{0}(x) & =x \\
y_{n+1}(x) & =-(x-1) \int_{0}^{1} \int_{0}^{s} y_{n}(u) d u d s \\
& -\int_{x}^{1} \int_{0}^{s} y_{n}(u) d u d s .
\end{aligned}
$$

Com isso é possível determinar as funções $y_{n}(x)$ começando por $y_{1}(x)$ que é dado por

$$
\begin{aligned}
y_{1}(x) & =-(x-1) \int_{0}^{1} \int_{0}^{s} y_{0}(u) d u d s-\int_{x}^{1} \int_{0}^{s} y_{0}(u) d u d s \\
& =-(x-1) \int_{0}^{1} \int_{0}^{s} u d u d s-\int_{x}^{1} \int_{0}^{s} u d u d s \\
& =-\frac{x}{6}+\frac{x^{3}}{6} .
\end{aligned}
$$

Agora a função $y_{2}(x)$ pode ser calculada como

$$
\begin{aligned}
y_{2}(x) & =-(x-1) \int_{0}^{1} \int_{0}^{s} y_{1}(u) d u d s-\int_{x}^{1} \int_{0}^{s} y_{1}(u) d u d s \\
& =-(x-1) \int_{0}^{1} \int_{0}^{s}\left(\frac{u^{3}}{6}-\frac{u}{6}\right) d u d s \\
& -\int_{x}^{1} \int_{0}^{s}\left(\frac{u^{3}}{6}-\frac{u}{6}\right) d u d s \\
& =\frac{7 x}{360}-\frac{x^{3}}{36}+\frac{x^{5}}{120} .
\end{aligned}
$$

Logo, para o quarto termo da série, tem-se

$$
\begin{aligned}
y_{3}(x) & =-(x-1) \int_{0}^{1} \int_{0}^{s} y_{2}(u) d u d s-\int_{x}^{1} \int_{0}^{s} y_{2}(u) d u d s \\
& =-(x-1) \int_{0}^{1} \int_{0}^{s}\left(\frac{7 u}{360}-\frac{u^{3}}{36}+\frac{u^{5}}{120}\right) d u d s \\
& -\int_{x}^{1} \int_{0}^{s}\left(\frac{7 u}{360}-\frac{u^{3}}{36}+\frac{u^{5}}{120}\right) d u d s \\
& =-\frac{31 x}{15120}+\frac{7 x^{3}}{2160}-\frac{x^{5}}{720}+\frac{x^{7}}{5040} .
\end{aligned}
$$

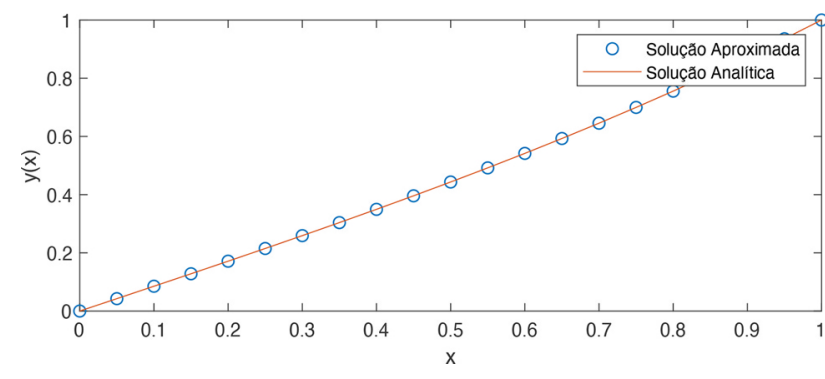

Figura 3: Comparação entre a solução analítica, equação 3.4 .2 , e a solução aproximada, equação 3.4 .13 , obtida através do método da decomposição de Adomian. A solução aproximada até a quarta parcela da série e mostra concordância com a solução analítica.

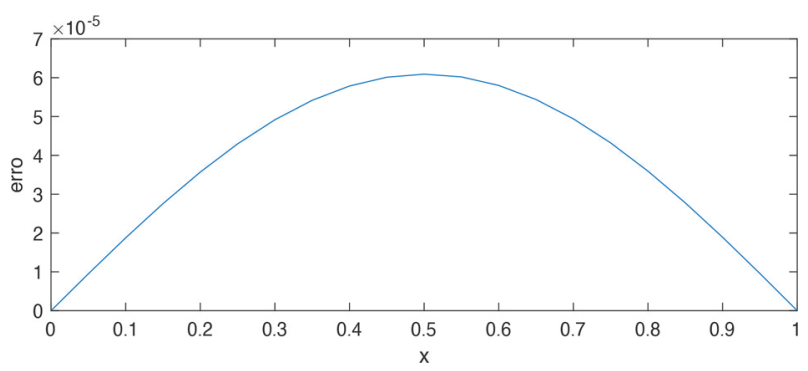

Figura 4: Módulo da diferença entre a solução analítica, equação 3.4 .2 , e a solução aproximada, equação 3.4.13. Observa-se que a solução aproximada tem quatro casas decimais de precisão apenas com quatro termos da série.

Portanto, a solução até a quarta parcela resultará em

$$
\begin{aligned}
& y(x)=y_{0}(x)+y_{1}(x)+y_{2}(x)+y_{3}(x)+\cdots \\
& =x+\left(-\frac{x}{6}+\frac{x^{3}}{6}\right)+\left(\frac{7 x}{360}-\frac{x^{3}}{36}+\frac{x^{5}}{120}\right) \\
& -\left(\frac{31 x}{15120}-\frac{7 x^{3}}{2160}+\frac{x^{5}}{720}-\frac{x^{7}}{5040}\right)+\cdots
\end{aligned}
$$

Nas figuras 3 e 4 são feitas comparações entre as soluções aproximada (equação (3.4.13) e analítica (equação (3.4.2) do problema. Verifica-se que a solução aproximada é capaz de representar muito bem a solução analítica apenas com quatro termos oferecendo erro absoluto inferior à $0,01 \%$. Mostrando, assim, a robustez do método da decomposição de Adomian também para encontrar soluções semianalíticas de problemas de valores de contorno.

Aqui se faz relevante constatar que todas as parcelas da série de solução encontradas satisfazem individualmente as condições de contorno $y(0)=0$ e $y(1)=1$. Este comportamento deve sempre ocorrer, independente se o problema é ou não-linear, e pode ser utilizado para verificar se as soluções parciais calculadas pelo método de Adomian encontram-se corretas 26$] 28]$. 


\section{Considerações Finais}

Neste trabalho foi apresentada uma revisão do Método da Decomposição de Adomian. Trata-se de um método adequado para se resolver equações diferenciais lineares e não-lineares, e tem sido aplicado sistematicamente em diversos problemas na literatura, em especial da física e engenharia. Neste sentido, foram trabalhados alguns exemplos envolvendo equações diferenciais ordináris e parciais, lineares e não-lineares e também problemas de valor inicial ou de contorno. Conforme destacado no trabalho, este método pode apresentar convergência muito rápida o que pode contrastar com outros métodos numéricos ou semi-analíticos rotineiramente utilizados. Além disso se destaca a sua simplicidade, sobretudo pelo fato dos cálculos realizados em cada iteração serem triviais. Finalmente, é um método que pode ser aplicado em diversos tipos de problemas lineares, não-lineares, de valor inicial, de contorno, de ordem fracionária e também algébricos sem grande necessidade de adaptação. Como perspectiva futura, vislumbramos a aplicação deste método em outras situações da física e da engenharia, bem como o desenvolvimento de um algoritmo computacional, utilizando aplicativos de matemática simbólica, para se obter soluções dos mais diversos tipos de problema e com precisão arbitrária.

\section{Referências}

[1] G. Adomian, Stochastics Systems (Academic Press, Cambridge, 1983).

[2] G. Adomian, J. Math. Anal. Appl. 135, 501 (1988).

[3] G. Adomian e R. Rach, Computers Math. Applic. 19, 9 (1990).

[4] G. Adomian e R. Rach, J. Math. Anal. 174, 118 (1993).

[5] G. Adomian, Solving Frontier Problems of Physics - The Decomposition Method (Springer, Berlin, 1994).

[6] A.M.A. El-Sayed e M. Gaberb, Phys. Lett. A 359, 3 (2006).

[7] O. González-Gaxiola e A. Biswasb, Optik 179, 434 (2019).

[8] E. Babolian e J. Biazarb, Appl. Math. Comput. 129, 339 (2002).

[9] F. Shakeri e M. Dehghan, Comput. Math. Appl. 54, 1197 (2007).

[10] R.K. Bhattacharyya e R.K. Bera, Appl. Math. Lett. 17, 703 (2004).

[11] N. Duan e K. Sun, IEEE Trans. Power Syst. 32, 430 (2017).

[12] S. Momani e Z. Odibat, Appl. Math. Comput. 177, 488 (2006).

[13] J. Muscat, Functional Analysis (Springer, Suiça, 2014).

[14] M.M. Hosseini e H. Nasabzadeh, Appl. Math. Comput. 82, 536 (2006).

[15] J.S. Duan e R. Rach, Comput. Math. Appl. 63, 1557 (2012).

[16] Y. Cherruault, Kybernetes 18, 31 (1989).

[17] Y. Cherruault, Math. Comput. Model. 14, 83 (1990).

[18] K. Abbaoui e Y. Cherruault, Math. Comput. Model. 28, 103 (1994).
[19] Y. Cherruault e G. Adomian, Comput. Math. Appl. 29, 103 (1995).

[20] I.L. El-Kalla, Appl. Math. Lett. 21, 372 (2007).

[21] A. Abdelrazec e D. Pelinovsky, Numer. Methods Partial Differential Eq. 27, 749 (2011).

[22] H. Zoubeir, arXiv:1903.07399 (2019).

[23] J.R. Taylor, Classical Mechanics (University Science Books, Mill Valley, 2005).

[24] A. Beléndez, C. Pascual, D.I. Méndez, T. Beléndez e C. Neipp, Rev. Bras. Ens. Fis. 29, 645 (2007).

[25] L.C. Evans, Partial Differential Equations (American Mathematical Society, Providence, 1998).

[26] B. Jang, J. Comput. Appl. Math. 219, 253 (2008)

[27] E.H. Aly, A. Ebaid, R. Rachc, Comput. Math. Appl. 63, 1056 (2012).

[28] J.S. Duan, R. Rach, A.M. Wazwaz, T. Chaolu e Z. Wanga, Appl. Math. Model. 37, 8687 (2013). 